\title{
Development of a technique for the real-time determination of crack geometries in laboratory samples
}

\author{
Thomas M Buss*, James P Rouse, Christopher J Hyde and Seamus D Garvey
}

University of Nottingham, University Park, Nottingham, NG7 2RD, UK.

\begin{abstract}
.
Crack size determination using electrical potentials both in service and in the laboratory has been undertaken for many years. In the laboratory this has mainly concentrated on the measurement of crack depth, with either alternating current (AC) or direct current (DC) supplies. Some work to determine the varying depth along the width of cracks as an inspection tool of in service parts using mapping methods has been done. This has used both $\mathrm{AC}$ and $\mathrm{DC}$ utilising various models to understand the data recorded, in Alternating Current Potential Drop (ACPD) a range of frequencies have been used to give various skin depths.
\end{abstract}

The resulting analyses have been grouped into two groups 'thin skin' and 'thick skin', in the thin skin case the skin depth is significantly smaller than the depth of the crack $1 / 10$ th of the crack depth whereas in the thick skin cases are for cases where skin depth is over this limit. Some work has been carried out to try and unify these two approaches.

The work presented here looks to develop a method using variable frequency ACPD to resolve further information about cracks growing in laboratory specimens. A system has been developed to rapidly sweep a wide frequency band and record voltage drop across a crack or feature. A selection of steel samples with known geometries and features have been used to trial and benchmark the technique. These samples have a range of cross sections as well as machined features or a range of shapes and sizes to simulate a range of crack geometries. This work has been approximated using a 2D computational model. This has been done using a reduced thickness approach.

\footnotetext{
Corresponding author: thomas.buss@,nottingham.ac.uk
} 


\section{Introduction}

Direct Current Potential Drop (DCPD) as well as ACPD are often used to monitor crack length in crack initiation and crack growth testing. For both techniques a constant current is injected into the sample. In DCPD the voltage measured across the crack increases with crack length due to the reduction in area of the remaining ligament. High frequency ACPD work utilises the skin effect, this means that the current flows in a thin skin along the surface of the sample, as the crack grows the path that the current flows along increases, this again leads to an increase in the voltage measured across the crack. A schematic representation of this can be seen in Figure 1.

It has been show that the thin skin only works where the crack is greater than ten times the skin depth, at intermediate frequencies neither of these situations are true. In the transitional frequencies there is significant error in both methods, some empirical work has been published bridging these two conditions[1]-[3].

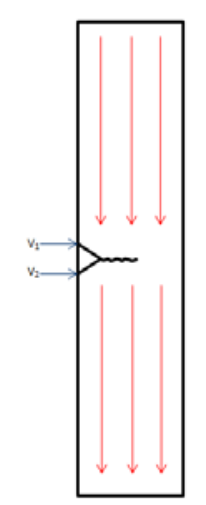

DCPD - Uniform Current Flow

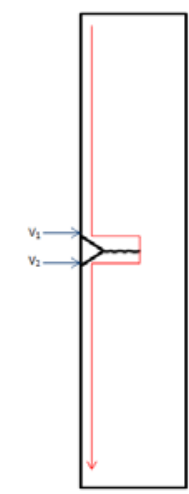

ACPD - Current Flowing on the Surface (Skin Effect)
Fig. 1. Current flow in DCPD and ACPD.

There has been a range of work concerning crack measurement on components using movable probes, this is termed Alternating Current Field Measurement (ACFM), but uses the same theories as ACPD but the movable probes can be used to map the response at different points along or across a crack[4]-[7].

This work summaries the current state of work being undertaken at the University of Nottingham, the aim of the work is to be able to monitor the shape of fatigue cracks growing in a range of fatigue conditions. Most importantly in Low Cycle Fatigue (LCF) and Thermomechanical Fatigue (TMF) where is has been shown that non-uniform crack growth can be prevalent and the measurement of these can be difficult.[8] Quite often efforts are made to stop tunnelling occurring in the test, by modified specimen geometries[9], interrupting the test to put different loading on the sample which has been shown to return the crack to a uniform shape or post processing to try to understand the stress intensity factor[10]. Current work however is not being undertaken in live crack growth experiments, efforts are concentrating on characterising response using a range of samples with known shape. The aim being to understand sample shape and the shape of features in samples by simple two point measurements, without the need for mapping the potentials over the surfaces. This is being undertaken by utilising a wide range of Alternating Current $(\mathrm{AC})$ frequencies and linking the results to the expected results generated from computational models. The work presented here looks at the first set of very simple samples. The samples chosen have identical cross sectional areas and do not contain any features or cracks, the only difference is the cross sectional shape. This has been done to enable the understanding of shape, the aim is to understand the fundamentals of the technique before embarking on more complex problems.

\section{Experimental}

Four samples have been manufactured with the same length $(55 \mathrm{~mm})$ and cross sectional area $\left(100 \mathrm{~mm}^{2}\right)$, but different cross section shapes. The four chosen are circular, hexagonal, square and triangular. The material used is 316 stainless steel. An image of the experimental setup can be seen in Figure 2.

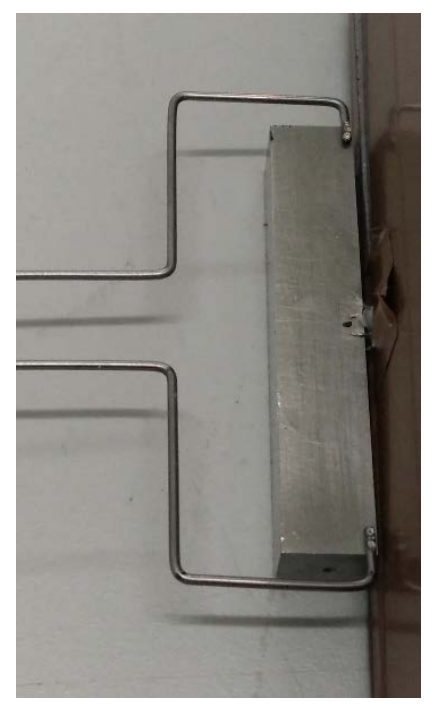

Fig. 2. Square Section Sample

\footnotetext{
Corresponding author: thomas.buss@nottingham.ac.uk
} 
A swept sine wave signal was used to sample frequencies from $15 \mathrm{~Hz}$ to $220 \mathrm{kHz}$ covering skin depths from quasi-DC to thin skin at $220 \mathrm{kHz}$. At $15 \mathrm{~Hz}$ the skin depth is approximately $28 \mathrm{~mm}$ which is larger than the width of any of the samples and at $220 \mathrm{kHz}$ the skin depth is $0.22 \mathrm{~mm}$ which falls in to the thin skin range[1], [11]. Throughout all the experiments the amplitude of the current injected was kept at 3 amps. The voltage between two points symmetrically arranged around the midpoint of the length of the samples was measured, for all sample geometries these were placed with a separation of $2 \mathrm{~mm}$ ( $1 \mathrm{~mm}$ either side of midpoint) and on the same apex as the current injection points. Induced voltages due to electromagnetic (EM) pickup were controlled by a repeatable current input path consisting of some custom made steel wires $1.14 \mathrm{~mm}$ in diameter, the shape and location of these could be easily controlled. These were connected to the current input lead by a screw terminal block and connected to the samples using spot welding. This enabled good and repeatable electrical connection for the input current.

The measurement wires were also shielded by the use of a die-cast box which can be seen on the right hand side of Figure 2 this was electrically insulated from the sample by means of a thin polymer tape. The box shielded the connection wires and the connector block from EM interference, once leaving the die cast box shielded instrument wire was used to continue the shielding to the amplifier. This enabled nearly the whole length of the measurement wires to be shielded from EM pickup. Repeat experiments were carried out to understand the level of repeatability of this setup, the data from two experiments on the square cross section sample can be seen in Figure 3, in these experiments all the wires were removed and new ones installed so this replicates a full 'new' test.

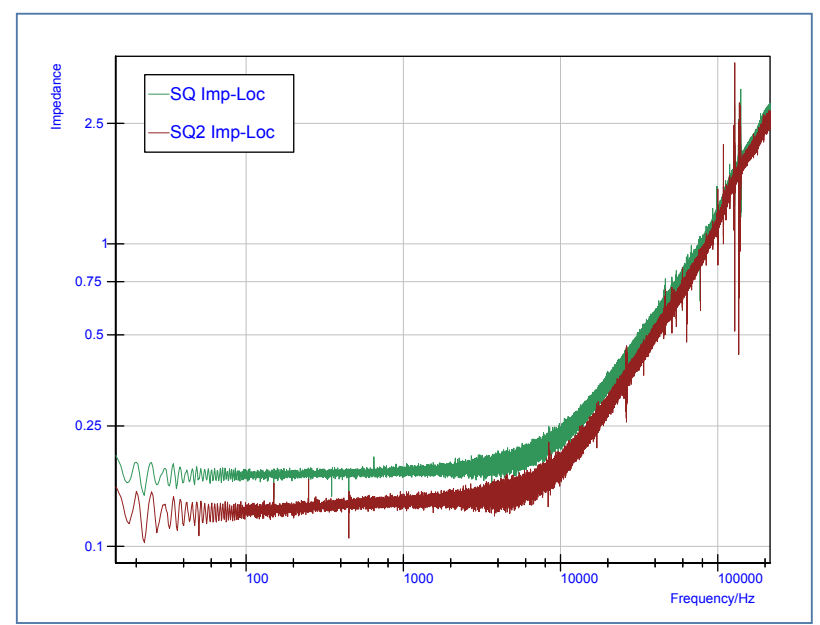

Fig. 3. Square Section Data
It can be seen in Figure 3 that there is an offset between the two curves across the frequency range. Due to the large skin depth at low frequencies the impedance at low frequencies if only governed by the cross sectional area and the path length. As the cross sectional area in all the samples is the same $\left(100 \mathrm{~mm}^{2}\right)$ the differences at low frequencies are due to variation in the experimental setup, the most significant variation is likely to be due to small variation in the spacing of the voltage measurement wires. Figure 3 only shows the square cross section result but this is also true for the other geometries, in the data processing this has been accounted for and the curves have all been shifted onto the same impedance as the circular cross section sample based on the readings at $100 \mathrm{~Hz}$ as the response at this frequency can be considered quasi-DC.

The input was generated using a piece of LabVIEW code that generated a swept sine signal this was output through an NI-PXIe-6124 input/output module. This signal was fed into a bespoke amplifier supplied by Fylde Electronics which amplifies the voltage supplied and outputs a current in real time that is proportional to the voltage input. The measured signals were fed back in to the same PXI unit. Due to the small magnitude of the voltages being measured the signals were amplified 1000 times using a Fylde FE-H793-TA before the PXI unit. The output data rate was $2.5 \times 10^{6}$ samples per second and the signals were read back at $4 \times 10^{6}$ samples per second. The data was processed using the National Instruments DIAdem software. The fast furrier transform was run on the signals and the root mean squared (RMS) amplitude of this was used to represent the magnitude of the signals in all these analyses. As the input sweep was logarithmic the RMS amplitude of the current diminished with time therefore it was necessary to calculate the impedance between the measurement points, $V_{1}$ and $V_{2}$ in Figure 1 . To do this the FFT RMS amplitude of the voltage between these points was divided by the FFT RMS amplitude of the input current. The resulting data for all the samples can be seen in Figure 4.

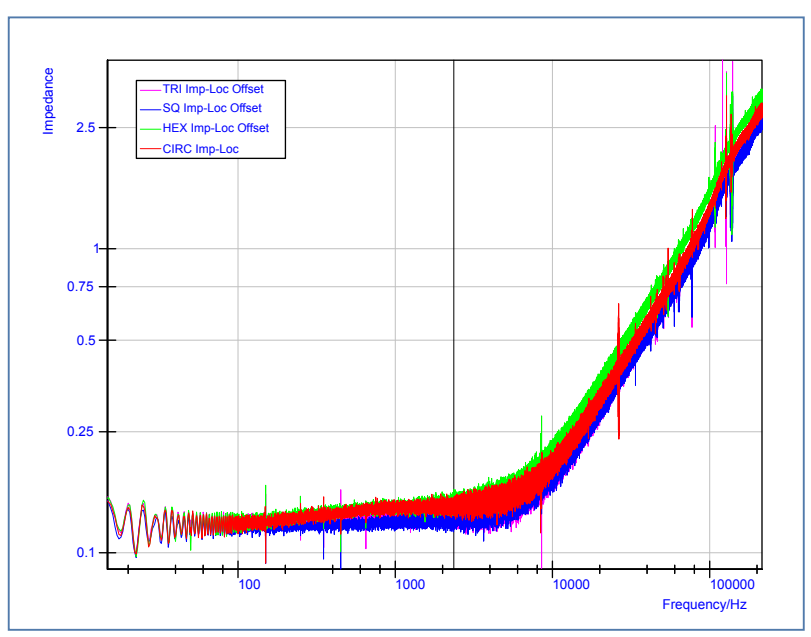

Fig. 4. Circular, Hexagonal, Square and Triangular Section Data 


\section{Modelling}

In this paper the theoretical analysis has been limited to a reduced thickness model. Given knowledge of the electrical and magnetic properties the skin depth at any given frequency can be calculated. If this is less than half the effective diameter of the samples this can be taken as the thickness of the conductor, this is termed a reduced thickness approach. This has been done in two ways, calculations of the resistance of the element between the measurement points and a computational model to simulate the voltages over the surface of the sample given the voltage input and a symmetry plain at the midpoint of the sample.

The calculations of the resistance between the two measurement points have been done for a number of frequencies. In this the conductor is considered to have a width equal to the perimeter of the sample and a thickness equal to the skin depth. Given this and the knowledge of the resistivity of the material, the resistance can be calculated by Equation 1, $\mathrm{x}$ being the distance between the measurement points and $\mathrm{A}$ the effective cross sectional area based on the perimeter of the sample and the skin depth at that frequency. This has been done for four frequencies across the range.

$$
R=\rho \frac{x}{A}
$$

The computational model was undertaken using a finite element technique in Abacus, images from this can be seen in Figure 5. As a DC problem is analogous to a heat transfer problem, the heat transfer functions have been used. This was set up as a shell model using DS4 elements the thickness of which were altered to mimic the skin depth at a range of frequencies. As mentioned above, only half of the sample has been modelled as the sample is symmetric about the centre line of the measurement points. The boundary conditions were set such that a point heat was positioned at the end of the sample on the same apex as the measurement points this is to simulate the input voltage and the face at the other end of the model, which is effectively the midline of the real sample, was set to zero temperature. The problem was solved for the steady state condition using the Abacus standard method.

The temperature at the point $1 \mathrm{~mm}$ from the 'zero end', on the same apex as this input heat, was calculated this can be considered as half the voltage between the measurement points and therefore can be compared to other results.

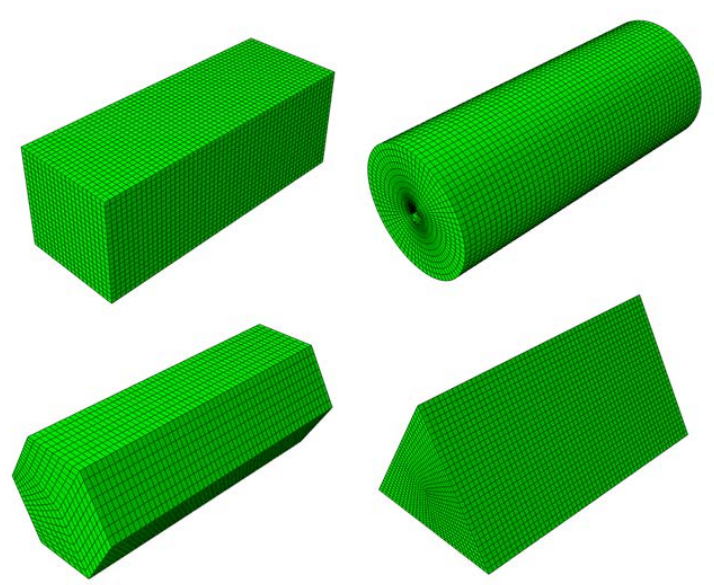

Fig. 5. FE Model

All the results both experimental and calculated have been normalised with respect to the low frequency quasiDC response to aid comparison. Plots of the results from the resistance calculations and heat flow model can be seen in Figure 6 and 7.

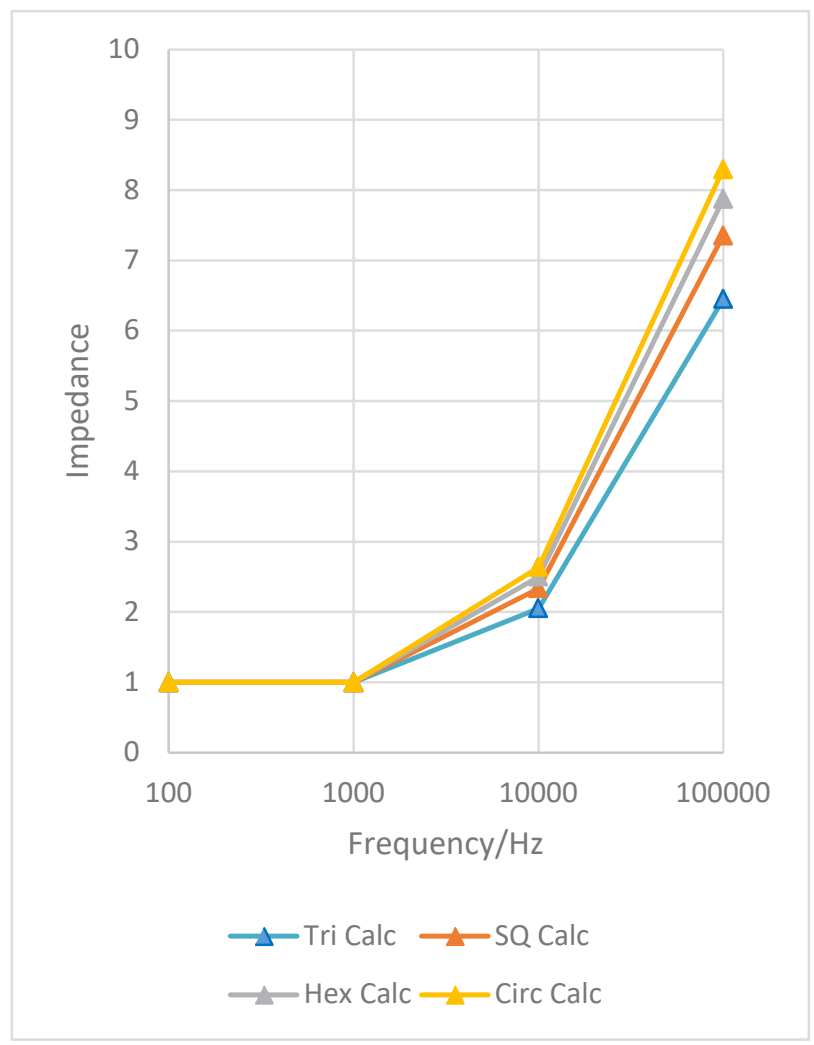

Fig. 6. Calculation Predictions 


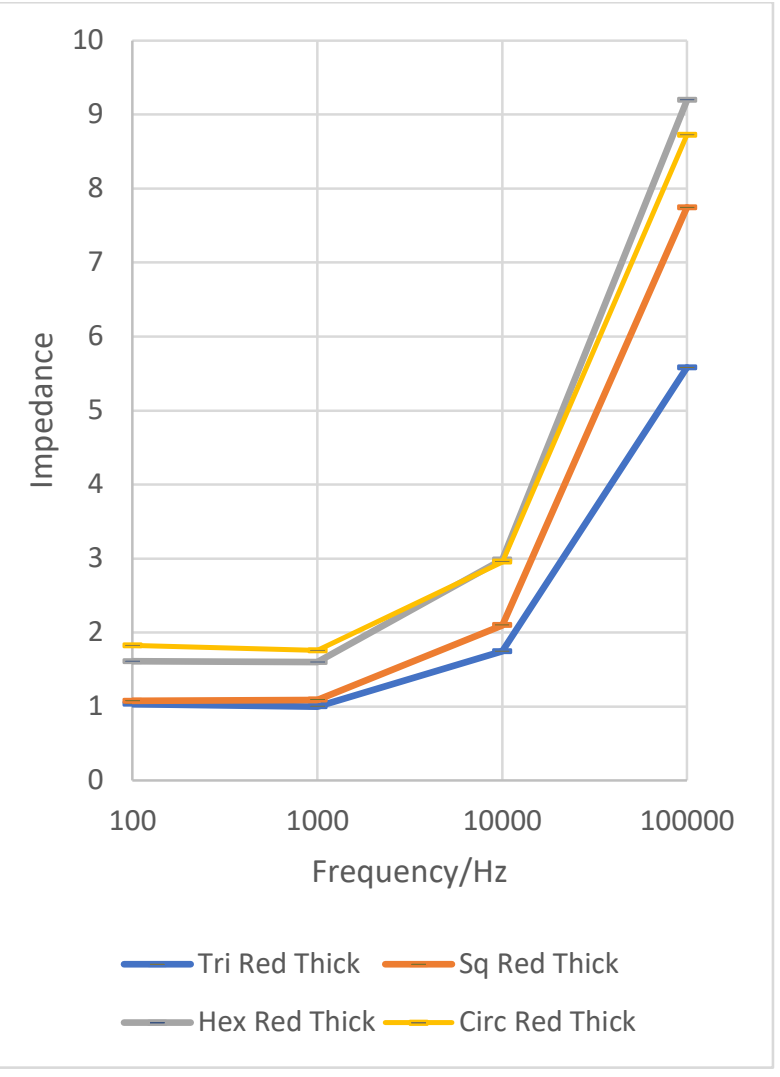

Fig. 7. Model Predictions

\section{Discussion}

It can be seen in the experimental data that the broad response for all the specimens follows the expected trends, at low frequency where the skin depth is greater than half the effective diameter the impedance is independent of frequency as the resistance is governed by the path length and real cross sectional area of the samples, therefore is constant. As the frequency rises the skin becomes less than the effective half the effective diameter and the impedance increases.

In this 316 stainless steel the skin depth reaches half the effective diameter at about $500 \mathrm{~Hz}$. It can be seen in the circular and hexagonal plots (Figure 8) that the impedance starts to rise at about this point, although does not immediately move onto the expected line for the thin skin assumption. This is less clear on the square and triangular cross section samples (Figure 9) and the impedance seems to remain flat until higher in the frequency range. It is likely that this is due to the hexagonal and circular samples being most similar to an idealised conductor, there are no sharp features to effect the distribution of the current in the intermediate skin thickness range. Whereas in the triangular and square samples the sharp corners will affect the current flow and this may be affecting the voltage measurement points in relation to the thinning skin.

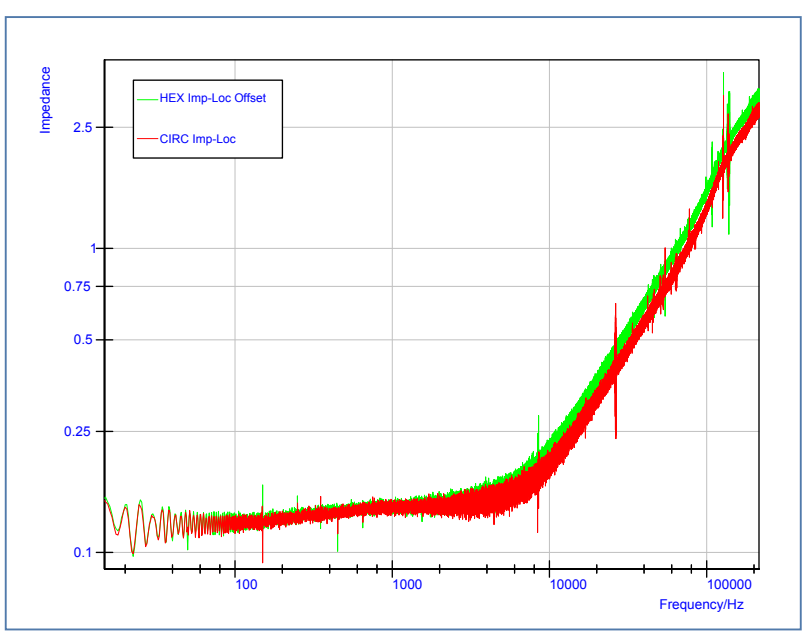

Fig. 8. Circular and Hexagonal Cross Sections Data

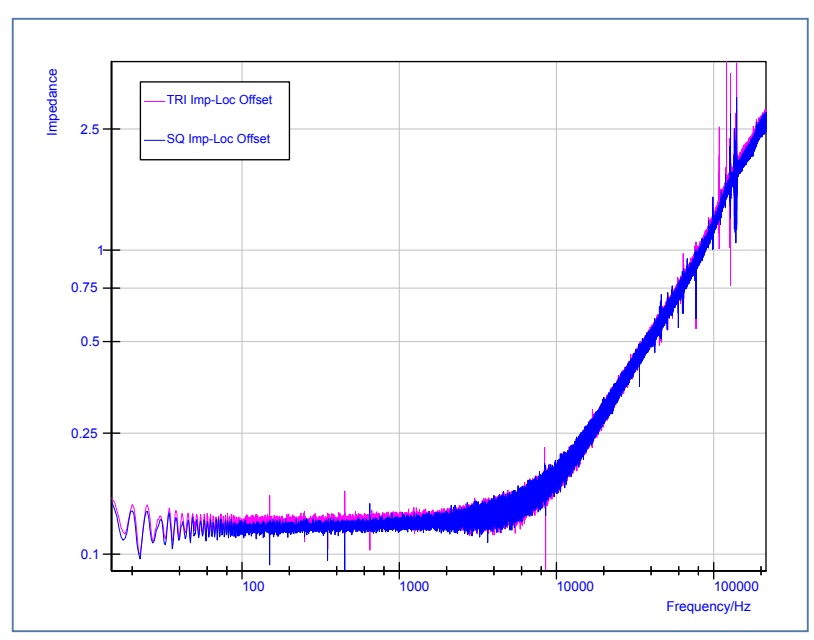

Fig. 9. Square and Triangular Cross Sections Data

In both the calculated impedance results and the Abacus model it can be seen that there is some reasonable correlation between the circular and hexagonal cross section samples, these giving the higher impedances and in the experimental results these two samples also give the highest impedances. In all the calculations and measured data the square and triangular section samples exhibit lower impedances although they match less well. This highlights some of the limitations of the current analytical approach.

It can also be seen that these theoretical methods are not sufficiently sensitive to pick up the differences highlighted above, at around $500 \mathrm{~Hz}$, between the circular and hexagonal when compared to the square and triangular samples. 


\section{Conclusions}

In samples of constant cross sectional area but with differing cross sectional shapes it can be seen that there are differences in impedances measured between two points centrally positioned on the test piece, as is customary in fatigue crack growth experiments. This has been considered theoretically using two methods. Calculations of resistance between the two measurement points and the results form a computational model based on voltages on a surface have been shown to correlate with the experimental results at lower frequencies, however deviations are significant at higher frequencies.

These theoretical techniques show some correlation to the experimental but also deviate in certain areas and lack some of the necessary sensitivity. There have been significant simplifications made in some of the calculations in relation to the current flow at the corners of the specimens. Also this approach, approximating the AC skin to a think DC conductor[7], does not have any capacity to account for phase shifting which may give further information on samples geometry and eventually crack shape.

It is therefore suggested that further simulation work is required to probe further the added information available in the $\mathrm{AC}$ phase data and a better understanding of current flows will also feed into the impedance analysis.

\section{References}

[1] H. Saguy and D. Rittel, "Bridging thin and thick skin solutions for alternating currents in cracked conductors," Appl. Phys. Lett., vol. 87, no. 8, pp. 68-70, 2005.

[2] H. Saguy and D. Rittel, “Alternating current flow in internally flawed conductors: A tomographic analysis," Appl. Phys. Lett., vol. 89, no. 9, pp. 214-216, 2006.

[3] H. Saguy and D. Rittel, "Flaw detection in metals by the ACPD technique: Theory and experiments," NDT E Int., vol. 40, no. 7, pp. 505-509, Oct. 2007.

[4] M. Akama and M. Saka, "Nondestructive sizing of a 3D surface crack generated in a railway component using closely coupled probes for direct-current potential drop technique," Eng. Fract. Mech., vol. 72, no. 2, pp. 319-334, Jan. 2005.

[5] Y. Hashimoto, Y. Urabe, S. Masamori, Y. Kamiwaki, and K. Baba, "Procedure of crack shape determination by reversing DC potential method," Nucl. Eng. Des., vol. 138, no. 3, pp. 259-268, Dec. 1992.

[6] G. Sposito, "Advances in Potential Drop Techniques for Non-distructive Testing," 